\title{
Senescence can explain microbial persistence
}

\author{
Correspondence \\ I. Klapper \\ klapper@math.montana.edu
}

Received 6 February 2007

Revised 29 June 2007

Accepted 9 July 2007
I. Klapper, ${ }^{1,2}$ P. Gilbert, ${ }^{3}$ B. P. Ayati, ${ }^{4}$ J. Dockery ${ }^{1,2}$ and P. S. Stewart ${ }^{2,5}$

${ }^{1}$ Department of Mathematical Sciences, Montana State University, Bozeman, MT 59717, USA

${ }^{2}$ Center for Biofilm Engineering, Montana State University, Bozeman, MT 59717, USA

${ }^{3}$ School of Pharmacy and Pharmaceutical Sciences, University of Manchester, Manchester, UK

${ }^{4}$ Department of Mathematics, Southern Methodist University, Dallas, TX 75205, USA

${ }^{5}$ Department of Chemical and Biological Engineering, Montana State University, Bozeman, MT 59717, USA

It has been known for many years that small fractions of persister cells resist killing in many bacterial colony-antimicrobial confrontations. These persisters are not believed to be mutants. Rather it has been hypothesized that they are phenotypic variants. Current models allow cells to switch in and out of the persister phenotype. Here, a different explanation is suggested for persistence, namely senescence. Using a mathematical model including age structure, it is shown that senescence provides a natural explanation for persistence-related phenomena, including the observations that the persister fraction depends on growth phase in batch culture and dilution rate in continuous culture.

\section{INTRODUCTION}

It has been observed (Balaban et al., 2004; Bigger, 1944; Gilbert et al., 1990; Greenwood \& O'Grady, 1970; Harrison et al., 2005; Keren et al., 2004b; McDermott, 1958; Moyed \& Bertrand, 1983; Sufya et al., 2003; Wiuff et al., 2005), dating to Bigger (1944), that many antimicrobials, while effective in reducing bacterial populations, are unable to eliminate them entirely, even with prolonged exposure. The surviving cells, called persisters, may be small in number - Bigger (1944) reported less than 100 persisters out of $2.5 \times 10^{7}$ cells of Staphylococcus pyogenes after exposure to penicillin in some cases, for example - but nevertheless are subsequently able to repopulate upon removal of the challenging agent (see Lewis, 2001 for a general discussion). This phenomenon has recently gained increased attention in the context of biofilms (Spoering \& Lewis, 2001) where the persisting cells have the added protection of a polymeric matrix, making them particularly dangerous (see, for example, the models of Roberts \& Stewart, 2004, 2005; Ayati \& Klapper, 2007). The protection of microbial populations by persistence, whether formed by senescence or some other mechanism, is expected to be enhanced in biofilms because of the propensity of biofilms to harbour slow-growing or nongrowing cells (Drury et al., 1993; Tijhuis et al., 1994; Okabe et al., 1997).

Persisters have a number of interesting characteristics:

- Upon reculturing, persister cells enable repopulation (see, for example, Balaban et al., 2004).

- Persisters do not pass their tolerance to their progeny, and progeny do not inherit any greater tendency to be persisters. That is, persisters do not appear to be genetic variants (see, for example, Keren et al., 2004a; Balaban et al., 2004).

- It is observed that persister cells apparently grow slowly or not at all in the presence of antimicrobial agent (see, for example, Balaban et al., 2004).

- Persister cells demonstrate tolerance upon exposure to multiple antimicrobial agents. That is, persister cells with respect to one antimicrobial agent can also be tolerant upon exposure to different antimicrobial agents. As an example, see Sufya et al. (2003), where survivors (from an Escherichia coli batch culture) of a tetracycline challenge were also tolerant to ciprofloxacin and quaternary ammonium compound.

- Bacterial cultures demonstrate biphasic killing patterns in response to antimicrobial challenge. It has been suggested that this plateauing is a consequence of the presence of persisters (see, for example, Balaban et al., 2004).

- In continuous culture experiments, persister fractions are observed to increase with decreasing dilution rates (Sufya et al., 2003).

- One of the more puzzling observations is that changes in the population fraction of persister cells are growthphase-dependent - generally, though not always, population increases do not occur until the later stages of the exponential phase or even until the stationary phase. For example, Keren et al. (2004a) observed an increasing ratio of persister to total cell numbers over time in E. coli, Pseudomonas aeruginosa 
and Staphylococcus aureus batch cultures. In Balaban et al. (2004) the authors went as far as to posit that differences in onset of persister cell increases between different strains of $E$. coli imply the existence of more than one type of persister.

It been suggested that persistence is a phenotypic phenomenon. Balaban et al. (2004), Roberts \& Stewart (2005) and Cogan (2006) have proposed models in which cells are able to switch in and out of a protected, slow- or non-growing persister state with probabilities that are dependent possibly on environmental conditions. In this paper, based on observations of microbial senescence (Ackermann et al., 2003; Barker \& Walmsley, 1999; Mortimer \& Johnston, 1959; Stewart et al., 2005), we instead propose an alternative simple mechanism that can explain all of the above-mentioned properties.

The standard view of microbial cell division, at least for symmetric dividers, has been that a given cell (the mother cell) splits into two essentially identical, youthful daughter cells. However, Stewart et al. (2005) demonstrate that, even in symmetric dividers, the mother cell retains its identity. That is, splitting is functionally asymmetric. During cell division the mother cell spawns one youthful daughter cell while itself remaining in the population, having aged in the process (Fig. 1). Stewart et al. (2005) have shown that the mother cell shows increasing senescence over the course of a number of cell divisions in the form of slowing growth rate.

In introducing this idea of senescence, we make only three essential assumptions:

(1) bacterial cells age [here age is based on senescence (see Fig. 1) rather than the traditional 0-1 cell cycle model, e.g. Webb (1989)];

(2) older cells are more tolerant than younger cells of antimicrobial challenge; and

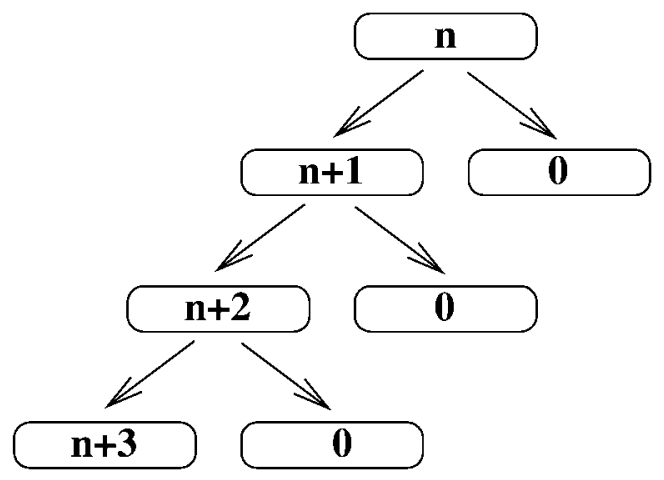

Fig. 1. A mother cell (top) divides at time intervals of, say, length 1. Upon each division, the mother cell has aged by one time unit and has produced a youthful daughter cell of age 0 .
(3) growth manifests as production of new cells. That is, upon division one progenic cell inherits the effects of age while the other does not (Fig. 1).

We thus regard older cells to be the persisters. In fact, one might well label senescent cells to be a separate persister phenotype (as opposed to a youthful phenotype), though we do not stress this interpretation.

The second assumption could be a consequence, for example, of decreased growth rate, but the particular mechanism does not really matter for our results. (The genetic bases for senescence and persistence are just beginning to emerge; see, for example, Nystrom, 2005; Vásquez-Laslop et al., 2006; Spoering et al., 2006.) In reference to the third assumption, we can interpret cell division as a new, youthful cell being born from an old one.

We make the following additional non-essential specific assumptions for definiteness:

(4) production rate of new cells decreases with age but remains greater than zero;

(5) cell death occurs at a constant rate;

(6) a given concentration of applied antimicrobial will kill cells of sufficiently young age, but will not affect older cells; and

(7) substrate usage depends on age and concentration, but in a separable way.

These extra assumptions matter in the details, but do not affect the qualitative results that we report with the exception that a non-zero growth rate (in assumption 4) is necessary in order to enable persister cells to repopulate after antimicrobial application. For consistency with the notion of decreasing activity, we suppose that older cells grow more slowly than younger cells, although this assumption is again not really necessary here; rather the focus is on senescence as a mechanism for tolerance.

\section{THEORY}

Microbial rates. See Table 1 for a listing of variables and parameters. We define $b(a, t) \Delta a$ to be the size of the bacterial population (in c.f.u.) between ages $a$ and $a+\Delta a$ at time $t$, and $c(t)$ to be the growth media concentration at time $t$. Following observations reported by Stewart et al. (2005), we suppose that cells senesce at a linear rate in time; other functional forms of age-increasing senescence could be used instead. In particular for a parameter $\lambda$ that we will call the senescence time, we define $r_{S}(a, c(t))$, the substrate usage rate per c.f.u. at time $t$ of cells of age $a$, by

$r_{S}(a, c(t))= \begin{cases}k_{S}[(1-a / \lambda)+\xi] c(t), & a \leq \lambda \\ k_{s} \xi c(t), & a>\lambda\end{cases}$

where $k_{S}$ is a first-order rate constant and $\xi$ is a base usage factor. 
Table 1. Variables, parameters and functions

\begin{tabular}{|c|c|c|c|}
\hline Symbol & Quantity & Units & Value \\
\hline$a$ & Age & $\mathrm{h}$ & \\
\hline$B(t)$ & Weighted population at time $t$ & c.f.u. & \\
\hline$b(a, t)$ & Population per age at age $a$ and time $t$ & c.f.u. $\mathrm{h}^{-1}$ & \\
\hline$b_{0}(a)$ & Initial population per age at age $a$ & c.f.u. $\mathrm{h}^{-1}$ & \\
\hline$C_{0}$ & Reservoir substrate concentration & $\mathrm{kg} \mathrm{m}^{-3}$ & Variable \\
\hline$c(t)$ & Substrate concentration at time $t$ & $\mathrm{~kg} \mathrm{~m}^{-3}$ & \\
\hline$c_{0}$ & Initial substrate concentration & $\mathrm{kg} \mathrm{m}^{-3}$ & 1 \\
\hline$D$ & Chemostat dilution rate & $\mathrm{h}^{-1}$ & Variable \\
\hline$d$ & Antimicrobial dosage & $\mathrm{kg} \mathrm{m}^{-3}$ & 0.01 \\
\hline$k_{d}$ & Nominal cell death coefficient & $\mathrm{h}^{-1}$ & 0.05 \\
\hline$k_{K}$ & Cell killing coefficient & $\mathrm{h}^{-1}$ & 10 \\
\hline$k_{S}$ & Substrate usage coefficient & $\mathrm{h}^{-1}$ c.f.u. $^{-1}$ & $10^{-6}$ \\
\hline$r_{K}(a, d)$ & Killing rate function & $\mathrm{h}^{-1}$ & \\
\hline$r_{S}(a, c)$ & Substrate usage rate function & $\mathrm{kg} \mathrm{m}^{-3} \mathrm{~h}^{-1}$ c.f.u. $^{-1}$ & \\
\hline$r_{X}(a, c)$ & Cell growth rate function & $\mathrm{h}^{-1}$ & \\
\hline$s(a)$ & Senescence factor & - & \\
\hline$t$ & Time & $\mathrm{h}$ & \\
\hline$v(s, c)$ & Senescence increase rate & Senescence $\mathrm{h}^{-1}$ & \\
\hline$Y_{S}$ & Cell yield coefficient & c.f.u. $\mathrm{m}^{3} \mathrm{~kg}^{-1}$ & $1.33 \times 10^{6}$ \\
\hline$\delta$ & Tolerance coefficient & $\mathrm{h} \mathrm{m}^{3} \mathrm{~kg}^{-1}$ & $1.5 \times 10^{3}$ \\
\hline$\lambda$ & Senescence time & $\mathrm{h}$ & 12 \\
\hline$\phi$ & Senescence proportionality constant & Senescence units & 0.75 \\
\hline$\sigma$ & Senescence & Senescence units & \\
\hline$\xi$ & Minimum substrate usage factor & - & $10^{-3}$ \\
\hline
\end{tabular}

Similarly, we define $r_{X}(a, c(t))$, the birth rate of new cells at time $t$ from cells of age $a$, by

$r_{X}(a, c(t))=Y_{S} r_{S}(a, c(t))$

where $Y_{S}$ is a yield coefficient. Additionally, according to assumption 5, the death rate function $r_{d}$ takes the form

$r_{d}=k_{d}$

where $k_{d}$ is a death rate constant. Finally, functional forms for antimicrobial application will be made below.

We stress that these choices are made for simplicity and for consistency with available data from the literature. The only essential condition we require is that the applied antimicrobial agent exhibit decreasing potency as cells age (see below). For example, age-dependence in $r_{S}$ and $r_{X}$ is unnecessary. Conversely, $r_{d}$ could be made functionally dependent, for example, on $a, c(t)$ or $\lambda$ if so desired.

Age structure. A mathematical description of age structure was first introduced by Lotka (1907) and McKendrick (1926), and many such representations have been used since. Here we define $b(a, t)$ to be the bacterial population density of age $a$ at time $t$. The equation governing $b$ is then

$\frac{\partial b}{\partial t}(a, t)+\frac{\partial b}{\partial a}(a, t)=-k_{d} b(a, t)$ where the term on the right-hand side reflects cell death. As previously mentioned, the death coefficient $k_{d}$ can be expected, in general, to depend on $c$ and $a$; we suppress such dependence here for simplicity as it does not affect our results in a qualitative way.

Equation (2) is valid for $0<a<\infty$. To obtain an equation for $b(a=0, t)$, i.e. for new cells at time $t$, we observe that such cells are 'born' at time $t$ from the existing population $b(a, t), a>0$. For example, the subpopulation of cells between ages $a$ and $a+\Delta a$ produces $r_{X}(a, c(t)) b(a, t) \Delta a$ new cells. Summing then over the entire existing population at time $t$, we obtain

$b(t, 0)=\int_{0}^{\infty} r_{X}(a, c(t)) b(a, t) d a$

A similar equation applies for the substrate concentration:

$\frac{d c}{d t}(t)=-\int_{0}^{\infty} r_{S}(a, c(t)) b(a, t) d a$

Equations (2)-(4) are supplemented by initial conditions $b(0, a)=b_{0}(a)$ for some supplied initial population distribution $b_{0}(a)$, and $c(0)=c_{0}$ for some supplied initial concentration $c_{0}$. For discussion of mathematical issues involved in age-differentiated systems such as the one considered here, see, for example, Cushing (1998) and Webb (1985). 
We write (see assumption 7) $r_{S}(a, t)=k_{S} s(a) c(t)$ where

$$
s(a)= \begin{cases}1-a / \lambda+\xi, & a \leq \lambda \\ \xi, & a>\lambda\end{cases}
$$

$(s(a)$ is the senescence factor) and so define a senescenceweighted total bacteria population

$B(t)=\int_{0}^{\infty} s(a) b(a, t) d a$

Then equations (2)-(4) become

$$
\begin{aligned}
& \frac{\partial b}{\partial t}+\frac{\partial b}{\partial a}=-k_{d} b, \quad a>0 \\
& b(t, 0)=Y_{S} k_{S} c B \\
& \frac{d c}{d t}=-k_{S} c B
\end{aligned}
$$

with initial conditions $b(0, a)=b_{0}(a), c(0)=c_{0}$. In the computations to follow we use $c_{0}=1 \mathrm{~kg} \mathrm{~m}^{-3}$ and

$$
b_{0}(a)= \begin{cases}\left(10^{2} \text { c.f.u. }\right) \lambda^{-1}(1-a / 2 \lambda), & a \leq 2 \lambda \\ 0 \text { c.f.u.h } & a>2 \lambda\end{cases}
$$

With this choice, the total initial population is $10^{2}$ c.f.u. distributed linearly in age over the age interval $(0,2 \lambda)$.

Senescence structure. In the previous section we have identified senescence with chronological age. In fact, Stewart et al. (2005) measure age in terms of cell divisions (as in Fig. 1), although in their experimental set up, cell division time and chronological time are approximately proportional. To allow for cell-division-based senescence, we consider a general senescence-structured population model,

$\frac{\partial}{\partial t} b(\sigma, t)+\frac{\partial}{\partial \sigma}(v(\sigma, c) b(\sigma, t))=-r_{d}(\sigma, c) b(\sigma, t), \sigma>t, t>0$

where $\sigma$ is an index of senescence and the function $v(\sigma, c)$ is the rate of increasing senescence per time. Senescence may be determined by chronological age (see previous section with $\sigma=a, v=1$ ) or in some other manner. In particular, we can identify senescence $\sigma$ to be proportional to the number of cell divisions as in Fig. 1 in which case $v(\sigma, t)=\phi r_{X}(\sigma, t)$ where $r_{X}$ is as given previously, see (1), and $\phi$ is a constant of proportionality.

For ease of calculations we change variables from $\sigma$ to $a$ to obtain

$$
\begin{aligned}
& \frac{\partial}{\partial t} b(a, t)+\frac{\partial}{\partial a} b(a, t)=-\widetilde{k}(\sigma, c) b(a, t) \\
& \frac{\partial}{\partial t} \sigma(a, t)=v(\sigma, c)
\end{aligned}
$$

where $a>0, t>0, \sigma(0, t)=0$, and $\widetilde{k}(\sigma, c)=k(\sigma, c)+\frac{\partial}{\partial \sigma} v(\sigma, c)$. The birth condition becomes

$b(0, t)=\int_{0}^{\infty} v(\sigma(a, t), c(t)) r_{X}(\sigma(a, t), c(t)) b(a, t) d a$
Chemostat model. In addition to batch culturing, we consider a chemostat system for which equations (5)-(7) become

$$
\begin{aligned}
& \frac{\partial b}{\partial t}+\frac{\partial b}{\partial a}=-\left(k_{d}+D\right) b, a>0 \\
& b(t, 0)=Y_{S} k_{S} c B \\
& \frac{d c}{d t}=-k_{S} c B+D\left(C_{0}-c\right)
\end{aligned}
$$

where $D$ is the chemostat dilution rate and $C_{0}$ is the reservoir substrate concentration.

Antimicrobial application. We include the effect of applied antimicrobial consistently with assumption (6): a given antimicrobial concentration $d$ applied at time $t$ to the bacteria population results in killing at rate $\gamma$ of sufficiently young (and hence susceptible) cells and does not affect older (and hence tolerant) cells. We assume for definiteness and consistency with our senescence assumption that tolerance age increases linearly with antimicrobial concentration. Other choices of age dependence, as long as they are monotone in senescence, can be made. While Stewart et al. (2005) report $1-2 \%$ decay in growth rate per generation, this is an average decay and presumably there is some distribution in senescence rate. This effect could be included in the model, but for the sake of simplicity we do not do so here. This omission might result in an overestimation of persistence numbers (perhaps only the most senescent outliers should be considered as persistent) but we do not believe there are other important qualitative consequences.

Then equation (5) is replaced by

$\frac{\partial b}{\partial t}+\frac{\partial b}{\partial a}=-\left(k_{D}+r_{K}(a, d)\right) b, a>0$

with killing rate function $r_{K}$ defined by

$r_{K}(a, d)= \begin{cases}k_{K}, & a \leq \delta d \\ 0, & a>\delta d\end{cases}$

where $k_{K}$ is a killing rate coefficient. (The corresponding change in equation (8) for the cell division version of senescence is similar.) Here, $\delta$ is an adjustable tolerance coefficient. For a given antimicrobial concentration $d$, a small $\delta$ means that cells become tolerant at a relatively young age, and a large $\delta$ means that cells become tolerant at a relatively old age. For numerical reasons, we slightly smooth the discontinuity in $r_{K}$ in the results reported below. Based on equation (15) then, for a given antimicrobial concentration $d$, cells of age $\delta d$ or greater result in persistence.

\section{METHODS}

Parameters The model we describe contains parameters $d, k_{d}, k_{S}, k_{K}$, $Y_{S}, \delta, \lambda, \phi$ and $\xi$ (plus two, $D$ and $C_{0}$ for the chemostat) (see Table 1). We chose representative, reasonable values (see below). Qualitative 
features are robust with respect to variation of these parameters within reasonable ranges. (The exception is $\delta$ for which an estimate is not available and for which variation changes the onset of persistence.) In fact, with the exception of $\delta$, the only essential imposed constraints on these parameters necessary for the results we report are that $k_{K}>k_{d}$, i.e. the antimicrobial killing rate is greater than the cell death rate, that $\delta>0$, i.e. cells become more tolerant with age, and that $\xi>0$ so that persister cells are capable of repopulation. We set the parameter values as follows.

Growth and substrate usage parameters. For definiteness, we set the length of the exponential phase to be approximately $10 \mathrm{~h}$ and the bacteria doubling time to be approximately $0.75 \mathrm{~h}$, resulting in approximately 13.3 doublings in the exponential phase. Given an initial value of $10^{2}$ c.f.u., we thus obtain approximately $10^{6}$ c.f.u. at the end of the exponential phase. These constraints require $c_{0} k_{S} \cong 2^{-13.3} \cong 10^{-6}$ and $c_{0} k_{S} Y_{S} \cong 1.33=(0.75)^{-1}$ (in units as listed in Table 1). We set $c_{0}=1 \mathrm{~kg} \mathrm{~m}^{-3}$ for initial substrate concentration, and then use $k_{S}=10^{-6} \mathrm{~h}^{-1}$ c.f.u. ${ }^{-1}, Y_{S}=1.33 \times 10^{6}$ c.f.u. $\mathrm{m}^{3} \mathrm{~kg}^{-1}$. (Roughly speaking, $k_{S}^{-1}$ fixes the population at the end of the exponential phase and $\left(k_{S} Y_{S}\right)^{-1}$ determines the length of the exponential phase via the doubling time.) The minimum substrate usage parameter $\xi$ is presumed to be small compared to 1 , and needs to be larger than zero in order for persister cells to repopulate. Otherwise its value is unimportant. We set $\xi=10^{-3}$. The value of the cell death rate is unimportant as well and could even be set to zero (the exception is for the continuous culture case where $k_{d}$ determines the slow dilution limit persister population); we use $k_{d}=0.05 \mathrm{~h}^{-1}$.

Senescence parameters. Supposing significant senescence after about 16 generations [Stewart et al. (2005) reports 1-2\% per generation] with a cell division time of $0.75 \mathrm{~h}$, we then obtain $\lambda=12 \mathrm{~h}$.

Antimicrobial parameters (except $\delta$ ). As a typical antimicrobial dosage, we use $d=0.01 \mathrm{~kg} \mathrm{~m}^{-3}$ (Roberts \& Stewart, 2005). As a typical antimicrobial killing rate we use $k_{K}=10 \mathrm{~h}^{-1}$ (Sufya et al., 2003).
Chemostat parameters. We allow $D$, the dilution rate, to vary. The other parameter, reservoir concentration $\left(C_{0}\right)$, does not affect our results and so it is arbitrary. To estimate $\phi$, we require, as above, significant senescence after 16 cell divisions, i.e. $\sigma$ should be approximately 12 after 16 cell divisions. Thus we set $\phi=12 / 16=0.75$.

The only important quantity without an estimate is the tolerance age $\delta d$ at which bacteria become tolerant to the antimicrobial [see equation (15)]. We assume that the tolerance age is equal to $\lambda$, the senescence time, and thus $\delta=\lambda / d=1.5 \times 10^{3} \mathrm{hr} \mathrm{m}^{3} \mathrm{~kg}^{-1}$. Increasing (decreasing) $\delta$ has the effect of increasing (decreasing) the tolerance age.

Numerical methods. Equation (14) (or equations (9)-(10) with accompanying conditions) along with equations (6) and (7) are solved numerically. We use a moving-grid Galerkin method in age with discontinuous piecewise linear functions post-processed to cubic splines for the approximation space in age (Ayati \& Dupont, 2002) along with a step-doubling method in time (Ayati \& Dupont, 2005). This combination was illustrated in Section 5, Ayati \& Dupont (2002), using the same code as used here.

\section{RESULTS}

In Fig. 2 we have applied an antimicrobial to a stationaryphase culture, Fig. 2(a), and to an exponential-phase culture, Fig. 2(b), and then 'reculture' afterwards by removing the antimicrobial and adding fresh medium. Note the biphasic survival in each case: there is an initial sharp die-off immediately after antimicrobial application followed by a second phase of die-off of older (persistent) cells due to natural causes. We also note that the number of persisters in this example does not grow substantially until the stationary phase - this is a consequence of the delay between birth of new cells in the exponential phase and (a)

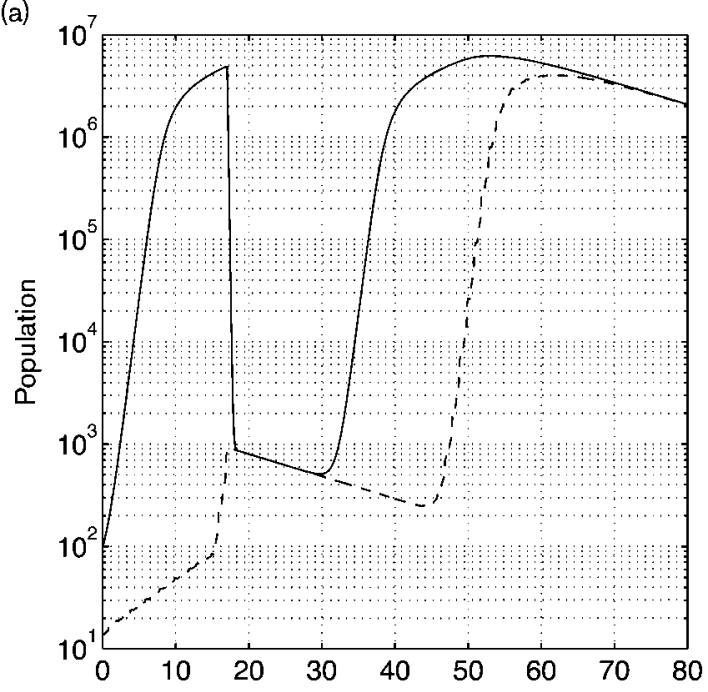

(b)

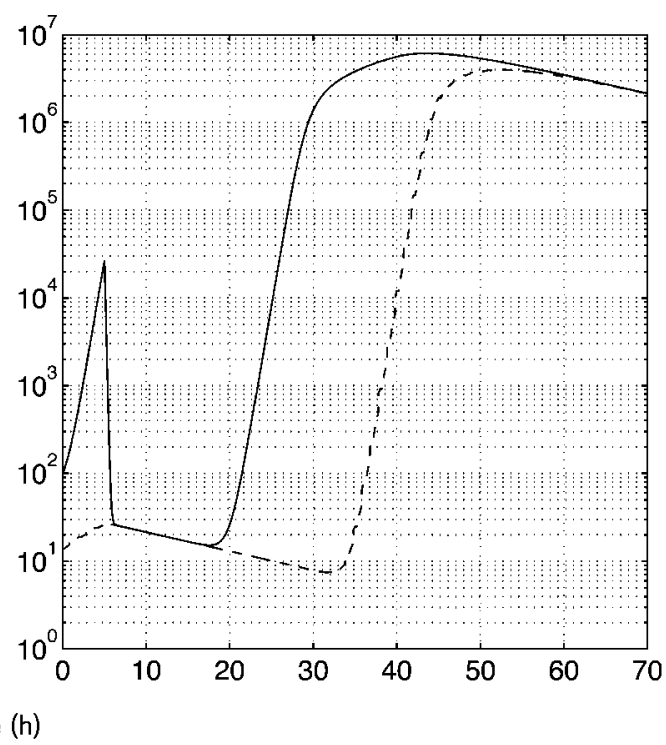

Fig. 2. Exposure of a batch culture to an antimicrobial with senescence determined by chronological age. (a) Antimicrobial applied during stationary phase at $t=17 \mathrm{~h}$ and removed at $t=27 \mathrm{~h}$, at which time the surviving cells are recultured at the $t=0 \mathrm{~h}$ nutrient level. (b) Antimicrobial applied during exponential phase at $t=5 \mathrm{~h}$ and removed at $t=15 \mathrm{~h}$, at which time the surviving cells are recultured at the $t=0 \mathrm{~h}$ nutrient level. Solid lines, all cells; dashed lines, persisters. 
ageing of those cells into persisters. That delay time depends on $\delta$, our only free coefficient. A small $\delta$ results in a short delay in the generation of persistent cells (i.e. growth in persister numbers even in the exponential phase) while a large $\delta$ results in a long delay in the generation of persistent cells. The growth at early times in persistence numbers seen in both Fig. 2(a) and Fig. 2(b) is solely a consequence of transients due to the initial age distribution in the inoculum. Note, for example, that persister numbers actually decline during the second exponential phase in both Fig. 2(a) and Fig. 2(b). We remark that the model predicts that most cells become persistent in late stationary phase. Late-stationary-phase data are scarce since all cells approach quiescence; however, data from continuous flow systems at low dilution rate (Sufya et al., 2003), which may be somewhat analogous to late-stationary-phase batch cultures, suggest that the persister percentage does indeed approach 100.

Fig. 3 illustrates the same computational experiment as in Fig. 2, but using cell-division-based senescence rather than chronological-age-based senescence. Note the similarity between the two. The onset of significant persistence is somewhat later in the cell-division-based case, but this is in fact an artefact of the choice of proportionality constant $\phi$ relating cell division rate to senesence rate $(\phi=0.75$ in Fig. 3). Large values of $\phi$ result in earlier onset. Due to the similarity of the results between chronological-age- and cell-division-based senescence models, we use only the somewhat simpler chronological age model below.
In Fig. 4 we present a computational version of the persister elimination experiment conducted by Keren $e t$ al. (2004a) (see Fig. 4 in that paper), qualitatively matching the results reported there. These authors observed that persister numbers could be driven downwards by frequent reculturing. In the present model, this phenomenon occurs because only a small percentage of cells are able to survive frequent cullings and thus reach senescence. In addition to serving as a comparison test of the persistence model experiment, our (computer) experiment serves to emphasize the point that the senescence mechanism presented here reproduces evidence that persisters are not formed in early exponential phase, and that by suppressing production of senescent cells, it is possible to suppress persister frequency as described by Keren et al. (2004a).

In fact, the persistence elimination experiment illustrated in Fig. 4 might be considered as an approximation to a chemostat with roughly $2 \mathrm{~h}$ turnover. So we also consider persister numbers in the chemostat system [equations (11)(13)] at steady-state (see Sufya et al., 2003). In particular, by setting $\partial b / \partial t=0$ in equations (11) and suppressing $t$ dependence, we obtain

$b(a)=b(0) e^{-\left(k_{d}+D\right) a}$

Thus the persister population fraction $P$ is given by

$P=\frac{\int_{\delta d}^{\infty} b(a) d a}{\int_{0}^{\infty} b(a) d a}=e^{-\left(k_{d}+D\right) \delta d}$ (a)

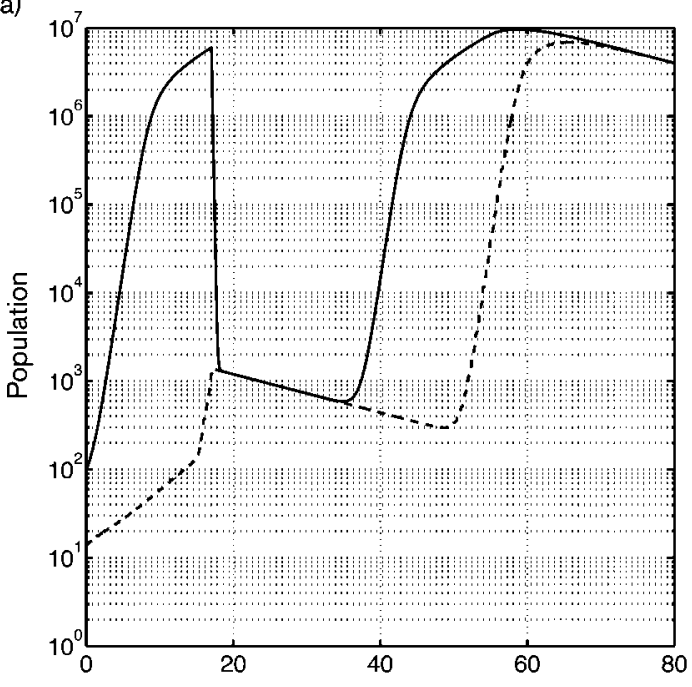

(b)

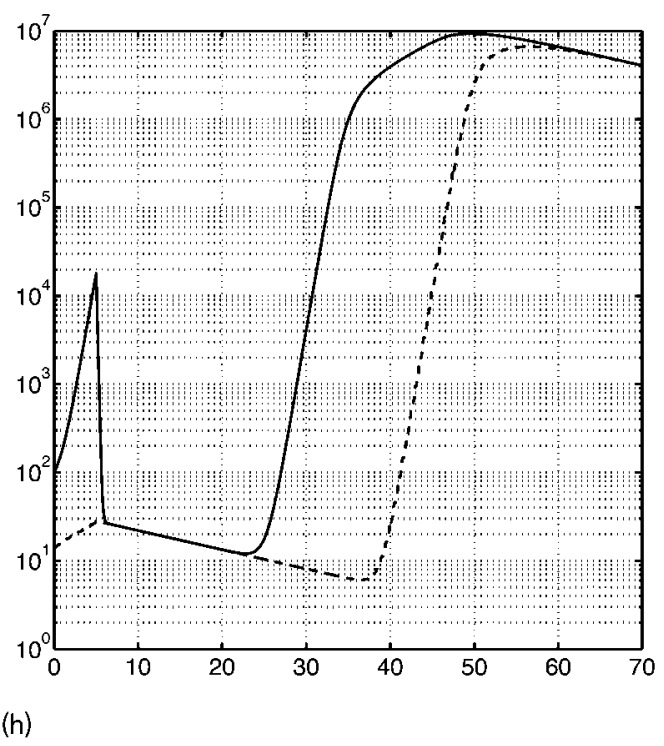

Fig. 3. Exposure of a batch culture to an antimicrobial as described in Fig. 2 except that senescence is proportional to cell division number rather than chronological age. (a) Antimicrobial applied during stationary phase at $t=17 \mathrm{~h}$ and removed at $t=27 \mathrm{~h}$, at which time the surviving cells are recultured at the $t=0 \mathrm{~h}$ nutrient level. (b) Antimicrobial applied during exponential phase at $t=5 \mathrm{~h}$ and removed at $t=15 \mathrm{~h}$, at which time the surviving cells are recultured at the $t=0 \mathrm{~h}$ nutrient level. Solid lines, all cells; dashed lines, persisters. 


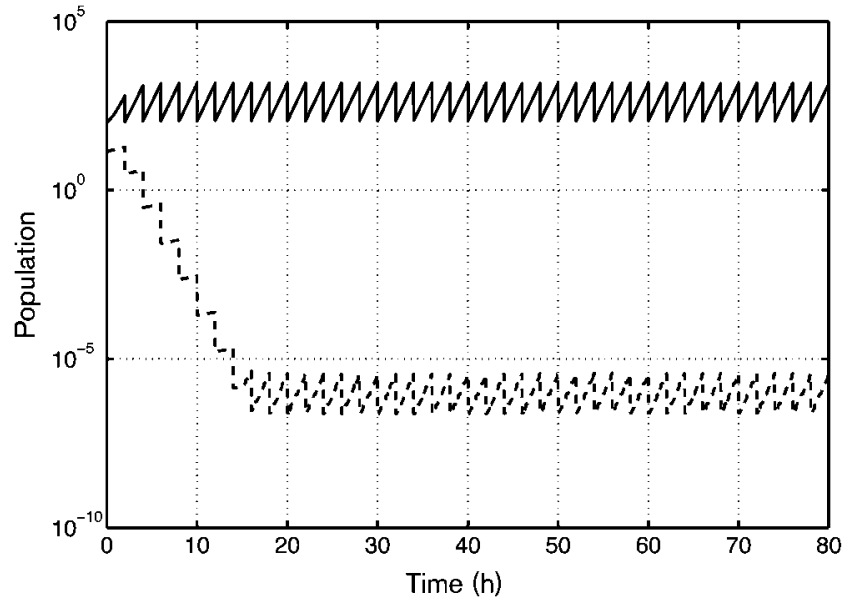

Fig. 4. Bacteria batch culture, diluted down to $10^{2}$ organisms every 2 h. Solid line, all cells; dashed line, persisters. See also Fig. 4 in Keren et al. (2004a).

See Fig. 5 (though at sufficiently high dilution rate, washout of cells exceeds the maximum specific growth rate and thus the biomass concentration, including persisters, becomes zero in steady-state). We again note the qualitative match to the experimentally reported results in Sufya et al. (2003). In particular, note the rapid transition from high to low persister fraction separating the two regimes when $D^{-1}$ is smaller than and larger than the persister age $\delta d$. For a small $D$, i.e. slow dilution, the persister fraction tends to a constant controlled by $k_{d}$, namely $e^{-k_{d} \delta d} \approx 0.55$ for the parameter values used here. For a large $D$, i.e. fast dilution, the persister fraction tends to zero. We remark that this characterization of persister fraction with respect to dilution rate (see Fig. 5) is independent of the details of substrate usage. That is, it is independent of equations (12) and (13). It is also independent of the form of the senescence factor.

\section{DISCUSSION}

The explanation of persistence as a symptom of senescence is an attractive one. Tolerance due to senescence, possibly because of reduced growth rate, explains in a simple manner the persistence characteristics listed in the Introduction:

- Offspring of old cells are of course young, and able to repopulate quickly.

- Offspring of old cells are young and active cells, and hence do not inherit low activity tolerance, i.e. they are not themselves persistent.

- Old cells grow slowly.

- Activity is independent of the choice of antimicrobial agent, so that inactive cells can demonstrate tolerance to multiple agents.

- In the presence of an antimicrobial agent, nonpersisters are killed quickly followed by slow die-off of persisters, i.e. biphasic behaviour occurs.

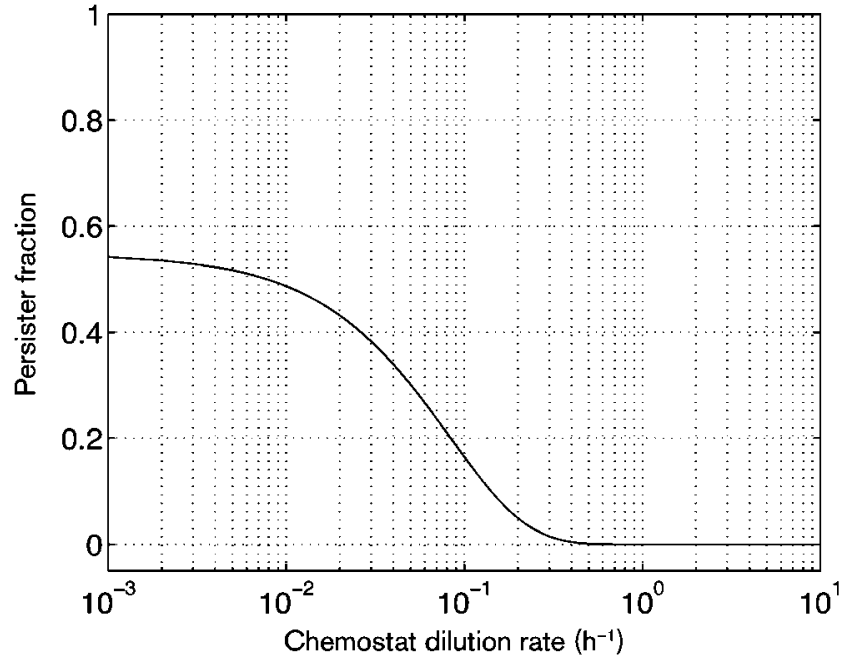

Fig. 5. Fraction of persisters in a steady-state chemostat as a function of chemostat dilution rate.

- Decreasing the dilution rate in a continuous culture allows more cells to age longer before washout, hence increasing the persister fraction.

- Increase in persistence numbers can appear to depend on growth phase. In particular new cells are, obviously, young cells and so exponential-phase growth does not affect persistence numbers until enough time has passed for those new cells to age sufficiently. Hence persistence numbers do not increase significantly until later in the exponential phase or even the stationary phase (and may actually decrease before).

All of these observations are qualitative properties of our model depending on assumptions (1)-(3) and, we believe, essentially independent of the particular choices made in the other assumptions.

A number of authors have previously suggested persisters to be switching phenotypic variants (e.g. Balaban et al., 2004; Cogan, 2006; Kussell et al., 2005; Roberts \& Stewart, 2005; Sufya et al., 2003; Wiuff et al., 2005), that is, that persisters are cells with the same genome but with different sets of genetic expression than 'normal' cells, and that a given cell can switch back and forth between the two states. The resulting phenotype switching model consists then of cells transiting between persister and non-persister phenotypes. We regard asymmetric ageing as an alternative pathway to the persistence phenomenon. Whereas the persister cell concept invokes, to many, binary switching or differentiation of cells between protected and non-protected states, the ageing concept in contrast posits a distribution of cell ages in a population and a correlation between age and susceptibility. Beyond thinking of ageing as a mechanism to generate persister cells, we suggest asymmetric ageing as a mechanism to generate distributed phenotypes (antimicrobial susceptibility or some other) within a population. 


\section{ACKNOWLEDGEMENTS}

I. K., J.D. and P.S.S. would like to acknowledge support from NIH award 5R01GM67245. B.P.A. would like to acknowledge support from NSF award DMS-0609854. I. K. and B. P. A. would like to thank IPAM, where much of this work was conducted, for its hospitality.

\section{REFERENCES}

Ackermann, M., Stearns, S. C. \& Jenal, U. (2003). Senescence in a bacterium with asymmetric division. Science 300, 1920.

Ayati, B. P. \& Dupont, T. F. (2002). Galerkin methods in age and space for a population model with nonlinear diffusion. SIAM J Numer Anal 40, 1064-1076.

Ayati, B. P. \& Dupont, T. F. (2005). Convergence of a step-doubling Galerkin method for parabolic problems. Math Comp 74, 1053-1065.

Ayati, B. P. \& Klapper, I. (2007). A multiscale model of biofilm as a senescence-structured fluid. Multiscale Model Simul 6, 347-365.

Balaban, N. Q., Merrin, J., Chait, R., Kowalik, L. \& Leibler, S. (2004). Bacterial persistence as a phenotypic switch. Science 305, 1622-1625.

Barker, M. G. \& Walmsley, R. M. (1999). Replicative ageing in the fission yeast, Schizosaccharomyces pombe. Yeast 15, 1511-1518.

Bigger, J. W. (1944). Treatment of staphylococcal infections with penicillin by intermittent sterilization. Lancet ii, 497-500.

Cogan, N. G. (2006). Effects of persister formation on bacterial response to dosing. J Theor Biol 238, 694-703.

Cushing, J. M. (1998). An Introduction to Structured Population Dynamics. Philadelphia: SIAM.

Drury, W. J., Stewart, P. S. \& Characklis, W. G. (1993). Transport of $1 \mu \mathrm{m}$ latex particles in Pseudomonas aeruginosa biofilms. Biotechnol Bioeng 42, 111-117.

Gilbert, P., Collier, P. J. \& Brown, M. R. W. (1990). Influence of growth rate on susceptibility to antimicrobial agents: biofilms, cell cycle, dormancy, and stringent response. Antimicrob Agents Chemother 34, 1865-1868.

Greenwood, D. \& O'Grady, F. (1970). Trimodal response of Escherichia coli and Proteus mirabilis to penicillins. Nature 228, 457-458.

Harrison, J. J., Ceri, H., Roper, N. J., Badry, E. A., Sproule, K. M. \& Turner, R. J. (2005). Persister cells mediate tolerance to metal oxyanions in Escherichia coli. Microbiology 151, 3181-3195.

Keren, I., Kaldalu, N., Spoering, A., Wang, Y. \& Lewis, K. (2004A). Persister cells and tolerance to antimicrobials. FEMS Microbiol Lett 230, 13-18.

Keren, I., Shah, D., Spoering, A., Kaldalu, N. \& Lewis, K. (2004B). Specialized persister cells and the mechanism of multidrug tolerance in Escherichia coli. J Bacteriol 186, 8172-8180.

Kussell, E., Kishony, R., Balaban, N. Q. \& Leibler, S. (2005). Bacterial persistence: a model of survival in changing environments. Genetics 169, 1807-1814.

Lewis, K. (2001). Riddle of biofilm resistance. Antimicrob Agents Chemother 45, 999-1007.
Lotka, A. J. (1907). Studies on the mode of growth of material aggregates. Am J Sci 24, 141-158.

McDermott, W. (1958). Microbial persistence. Yale J Biol Med 30, 257-291.

McKendrick, A. G. (1926). Applications of mathematics to medical problems. Proc Edin Math Soc 44, 98-130.

Mortimer, R. K. \& Johnston, J. R. (1959). Life span of individual yeast cells. Nature 183, 1751-1752.

Moyed, H. S. \& Bertrand, K. P. (1983). hipA, a newly recognized gene of Escherichia coli K-12 that affects frequency of persistence after inhibition of murein synthesis. J Bacteriol 155, 768-775.

Nystrom, T. (2005). Bacterial senescence, programmed death, and premeditated sterility. ASM News 71, 363-369.

Okabe, S., Yasuda, T. \& Watanabe, Y. (1997). Uptake and release of inert fluorescence particles by mixed population biofilms. Biotechnol Bioeng 53, 459-469.

Roberts, M. E. \& Stewart, P. S. (2004). Modeling antibiotic tolerance in biofilms by accounting for nutrient limitation. Antimicrob Agents Chemother 48, 48-52.

Roberts, M. E. \& Stewart, P. S. (2005). Modelling protection from antimicrobial agents in biofilms through the formation of persister cells. Microbiology 151, 75-80.

Spoering, A. L. \& Lewis, K. (2001). Biofilms and planktonic cells of Pseudomonas aeruginosa have similar resistance to killing by antimicrobials. J Bacteriol 183, 6746-6751.

Spoering, A. L., Vulic, M. \& Lewis, K. (2006). $g l p D$ and $p l s B$ participate in persister cell formation in Escherichia coli. J Bacteriol 188, 5136-5144.

Stewart, E. J., Madden, R., Paul, G. \& Taddei, F. (2005). Aging and death in an organism that reproduces by morphologically symmetric division. PLoS Biol 3, e45.

Sufya, N., Allison, D. G. \& Gilbert, P. (2003). Clonal variation in maximum specific growth rate and susceptibility towards antimicrobials. J Appl Microbiol 95, 1261-1267.

Tijhuis, L., van Benthum, W. A. J., van Loosdrecht, M. C. M. \& Heijnen, J. (1994). Solids retention time in spherical biofilms in a biofilm airlift suspension reactor. Biotechnol Bioeng 44, 867-879.

Vázquez-Laslop, N., Lee, H. \& Neyfakh, A. A. (2006). Increased persistence in Escherichia coli caused by controlled expression of toxins and other unrelated proteins. J Bacteriol 188, 3493-3497.

Webb, G. F. (1985). Theory of Nonlinear Age-Dependent Population Dynamics. New York: Marcel Dekker.

Webb, G. F. (1989). Alpha and beta curves, sister-sister and motherdaughter correlations in cell population dynamics. Comput Math Appl 18, 973-984.

Wiuff, C., Zappala, R. M., Regoes, R. R., Garner, K. N., Baquero, F. \& Levin, B. R. (2005). Phenotypic tolerance: antibiotic enrichment of noninherited resistance in bacterial populations. Antimicrob Agents Chemother 49, 1483-1494.

Edited by: C. Picioreanu 